\title{
An underlying prognosis predictor of hepatocellular carcinoma: Oncoprotein 18
}

\author{
SHU GONG, ZHONGHUA TAO, XIAOYAN LIU and LIN GAN \\ Research Centre for Preclinical Medicine, Luzhou Medical College, Luzhou, Sichuan 646000, P.R. China
}

Received September 02, 2013; Accepted October 21, 2013

DOI: 10.3892/br.2013.197

\begin{abstract}
Recent studies have reported the association between the expression of oncoprotein 18 (op18) and hepatocellular carcinoma (HCC). However, any underlying mechanistic connection between op18 expression and hepatocarcinogenesis is poorly understood. In the present study, Flag-pcDNA3.1 vector and Flag-pcDNA3.1-op18 plasmid were stably transfected in SMMC7721 cells, respectively. Stable SMMC7721 control and op18 overexpression SMMC7721 cell lines were constructed and identified by western blot analysis. Using a cell counting kit-8 (CCK8), it was shown that cell proliferation was significantly increased in the op18 overexpression SMMC7721 cell group $(0.60 \pm 0.05)$, compared with the control group $(0.29 \pm 0.03)$ at an absorbance of $450 \mathrm{~nm}(\mathrm{P}<0.01)$. Flow cytometry was used to analyze cell apoptosis by FITC-Annexin V and propidium iodide (PI) apoptosis assay kit. The results demonstrated that the percentage of apoptotic cells was inhibited to $5.80 \pm 0.33 \%$ in the op18 overexpression group, compared with $11.79 \pm 1.09 \%$ in the control group. Using FACS, single cell analysis data showed that op18 overexpression induced cell cycle arrest by inhibiting progression from $\mathrm{G} 2$ to $\mathrm{M}$ phase. The results suggest that op18 expression is closely associated with SMMC7721 cell proliferation and apoptosis, which appears to be a potential predictor of prognosis in HCC.
\end{abstract}

\section{Introduction}

Hepatocellular carcinoma (HCC) is the most common primary malignant liver tumor and ranks the fifth most prevalent malignant tumor worldwide. In addition, China has the highest incidence of HCC, accounting for $55 \%$ of all new cases globally. Although many risk factors, such as alcohol, aflatoxin, hepatitis, genetic predisposition, obesity and diabetes have been identified, the exact molecular mechanism has yet to be determined (1-3).

Correspondence to: Professor Lin Gan, Research Centre for Preclinical Medicine, Luzhou Medical College, 3-319 Zhongshan Road, Luzhou, Sichuan 646000, P.R. China

E-mail: gl-gump@163.com

Key words: oncoprotein 18, hepatocellular carcinoma, proliferation, apoptosis
Oncoprotein 18 (op18), also known as op17, op19 and stathmin, a ubiquitous 19-kDa cytosolic phosphoprotein, has been reported to play a critical role in microtubulin destabilizing, spindle assembly, chromosomal stability, cell shape, mitosis, and other cell processes. It has been reported that op18 expression correlates with tumorigenesis and tumor progression (4-7). However, few studies have directly compared cell proliferation and apoptosis in HCC cell lines transfected with op18. The mechanism of op18 in HCC should be elucidated as well as the significance of the upregulated expression of op18 in HCC cell lines. We therefore analyzed cell proliferation, apoptosis and cell cycle in op18 overexpression SMMC7721 cells by stably transfecting Flag-pcDNA3.1-op18 plasmid, compared with control SMMC7721 cells, which were transfected with Flag-pcDNA3.1 vector. The aim of this study was to examine the involvement of op18 in human hepatocarcinogenesis and to evaluate its prognostic significance in HCC.

\section{Materials and methods}

Cell lines and plasmids. Human HCC SMMC7721 cells were cultured in DMEM supplemented with $10 \%$ fetal bovine serum (Gibco, Carlsbad, CA, USA) at $37^{\circ} \mathrm{C}$ in a humidified atmosphere of 5\% $\mathrm{CO}_{2}$. The Flag-pcDNA3.1-op18 plasmid was kindly provided by Dr Baldassarre (Texas University, USA).

Stable transfection. The Flag-pcDNA3.1-op18 plasmid or Flag-pcDNA3.1 vector was transfected into SMMC7721 cells with Lipofectamine ${ }^{\mathrm{TM}} 2000$ (Invitrogen, Carlsbad, CA, USA) according to the manufacturer's instructions. Promycin (200 $\mu \mathrm{g} / \mathrm{ml})$ was used to select stably transfected cell lines. The transfection efficiency was then determined by western blot analysis.

Western blot analysis. Western blot analysis was performed as described previously (8). Briefly, total cell proteins were extracted from SMMC7721-op18 and control cells with cell lysis buffer (Beyotime, Jiangsu, China), separated on $10 \%$ SDS-PAGE gels and transferred onto PVDF membrane (Millipore, Billerica, MA, USA). The primary antibody (1:10,000; Abcam, Cambridge, MA, USA) was then added at $4^{\circ} \mathrm{C}$ overnight and bound with HRP-conjugated secondary antibodies at room temperature for $1 \mathrm{~h}$. Chemiluminescent signaling was detected using an ECL kit (Millipore) and autoradiography. 
A

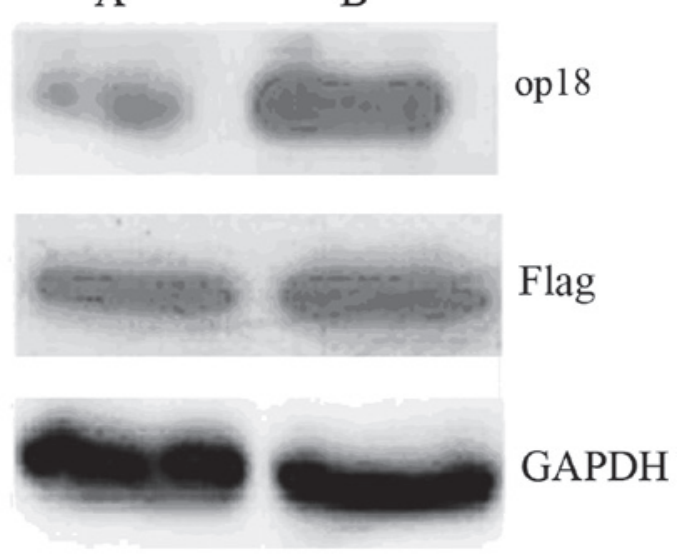

Figure 1. Western blot analysis for expression of oncoprotein 18 (op18) and Flag in the two cell groups. (A) The control group; (B) the op18 overexpression group. GAPDH was detected to normalize the expression of op18.

Cell proliferation assay. The cell counting kit-8 (CCK-8; Dojindo, Kumamoto, Japan) colorimetric assay was used to measure cell proliferation and viability with triplicate experiments for each set of conditions. SMMC7721 control and SMMC7721-op18 cells were seeded in 96-well plates at a density of $5 \times 10^{3}$ cells per well. The cells were cultured for $24 \mathrm{~h}$, the supernatant was removed, and $100 \mu 1$ of DMEM medium containing $10 \mu \mathrm{l}$ of CCK8 was added to each well for $1 \mathrm{~h}$ at $37^{\circ} \mathrm{C}$. The absorbance at $450 \mathrm{~nm}$ was measured with a plate reader (Multiskan GO Microplate Spectrophotometer; Thermo Fisher Scientific, Inc., Waltham, MA, USA).

Cell cycle assay. Cells pellet were digested and collected by trypsin, fixed in $70 \%$ ethanol on ice and then stained with $50 \mu \mathrm{g} / \mathrm{ml}$ propidium iodide (PI) (Sigma, St. Louis, MO, USA) and $0.1 \mu \mathrm{g} / \mathrm{ml}$ RNase A (Sigma). The cells were detected with flow cytometry using FACStar Plus (FACSCalibur Flow cytometer; Becton-Dickinson, Mountain View, CA, USA). Cell Quest software (BD CellQuest Pro Software, BD Biosciences, San Diego, CA, USA) was used to analyze the percentage of the cell population in each phase.

Cellular apoptosis assay. Apoptosis was assessed with the Annexin V-FITC kit according to the manufacturer's instructions. The cells were washed twice with cold PBS, digested, collected, and resuspended to binding buffer. Annexin V-FITC and PI were added (BioVision, Milpitas, CA, USA), and the cells were incubated for $10 \mathrm{~min}$ at room temperature in the dark. Then, $200 \mu \mathrm{l}$ binding buffer was added, and the cells were calculated with flow cytometry (FACScan, BD, Germany). The percentage of apoptosis was analyzed using the equation: $100 \mathrm{x}$ [experimental apoptosis (\%) - spontaneous apoptosis (\%)]/[100 - spontaneous apoptosis (\%)].

Statistical analysis. Results are expressed as means \pm SD of multiple experiments. Statistical analysis was performed with the Student's t-test for comparison between two groups or an analysis of variance (ANOVA) followed by Tukey's t-test for comparison of multiple groups. $\mathrm{P}<0.05$ was considered to indicate a statistically significant difference.

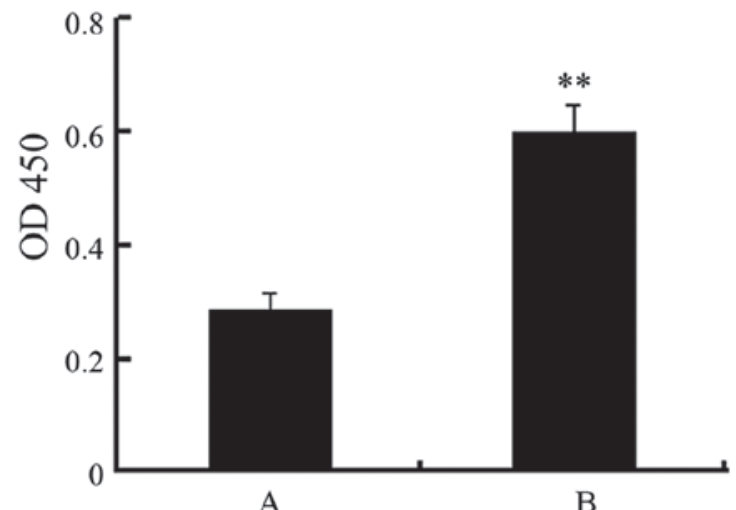

Figure 2. Cell proliferation was increased by oncoprotein 18 (op18) overexpression. Cell viability was evaluated by using the CCK8 assay following op18 upregulation. The data shown are from three independent experiments, ${ }^{* *} \mathrm{P}<0.01$. (A) The control group; (B) the op18 overexpression group.

A
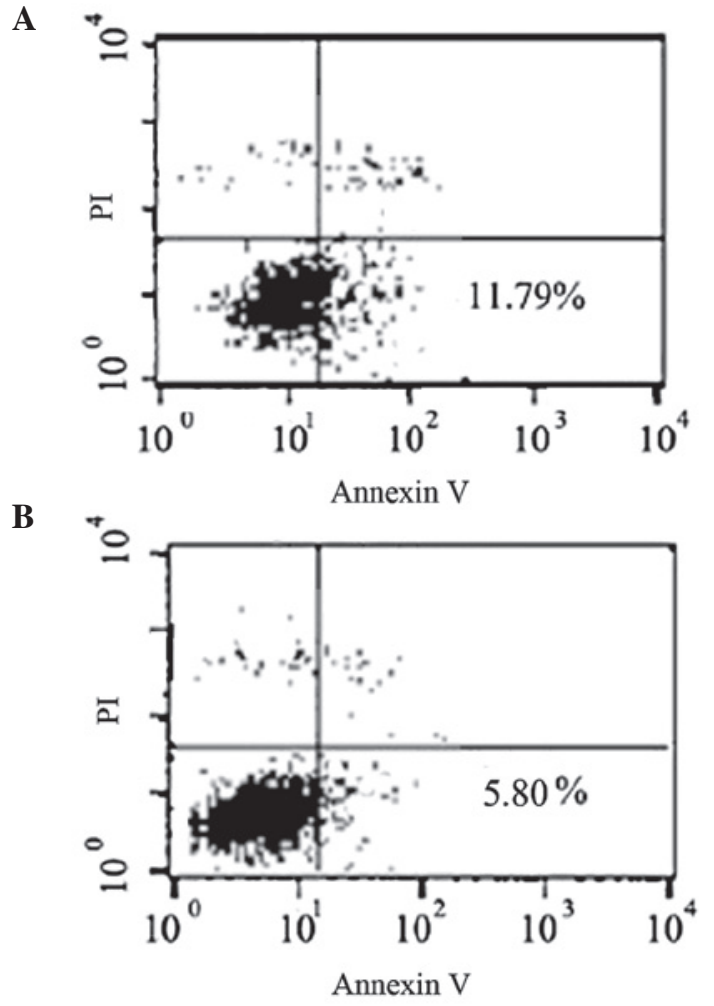

Figure 3. SMMC7721 apoptosis was inhibited by regulation of oncoprotein 18 (op18) expression. op18 overexpression suppressed cell apoptosis in the op18 overexpression group in SMMC7721 cells. Cells were stained with Annexin V (x-axis) and PI (y-axis) to quantify apoptosis and necrosis, respectively. (A) The control group; (B) the op18 overexpression group.

\section{Results}

Establishment and identification of op18 overexpression SMMC7721 stable cell lines. Stable transfection method was used to establish stably expressed Flag-pcDNA3.1 vector and Flag-pcDNA3.1-op18 plasmid human hepatocarcinoma SMMC7721 cell lines. Western blot analysis results revealed that op18 expression was increased in SMMC7721-op18 cells transfected with Flag-pcDNA3.1-op18 plasmid, compared 
with control SMMC7721-transfected Flag-pcDNA3.1 plasmid (Fig. 1).

Increase of SMMC7721 cell proliferation by op18 overexpression. To determine the effect of the upregulation of op18 expression on SMMC7721 cell proliferation, CCK8 assay was used to analyze cell proliferation. The results showed that cell proliferation was significantly increased in the op 18 overexpression SMMC7721 cell group $(0.60 \pm 0.05)$, compared with the control group $(0.29 \pm 0.03)$ at the absorbance of $450 \mathrm{~nm}$ ( $\mathrm{P}<0.01$, Fig. 2).

Inhibition of SMMC7721 apoptosis by upregulating op18 expression. Flow cytometry was performed to test the effect of op18 overexpression on SMMC7721 cell apoptosis through FITC-Annexin V and PI labeling. The results revealed that the percentage of apoptotic cells was inhibited to $5.80 \pm 0.33 \%$ in the op18 overexpression group, compared with $11.79 \pm 1.09 \%$ in the control group (Fig. 3).

SMMC7721 cell cycle arrested at the phase of G2/M by upregulation of op18 expression. To determine whether op18 plays a key role in the progression of SMMC7721 cell cycle, we demonstrated the effect of op18 overexpression in SMMC7721 cells on the cell cycle by single cell analysis using FACS. The results showed that op18 overexpression induced cell cycle arrest by inhibiting progression from $\mathrm{G} 2$ to $\mathrm{M}$ phase.

\section{Discussion}

op18 plays a crucial role in tumorigenesis and metastasis. Numerous studies have demonstrated the upregulated expression of op18 in several types of cancer, including breast, prostate, lung, ovarian cancer and sarcoma (9-11), which was associated with the malignant biological behavior of cancer cells, as well as a potential predictor of worse prognosis and poor treatment.

Recent studies have reported the association between the expression of op18 and HCC (12-14). Wang et al (12) found that op18 distinctly expressed proteins identified in HCC cells treated by gambogic acid (GA) by proteomic approach and western blotting. Furthermore, it was reported that the overexpression of op18 in HCC cells decreased their sensitivity, whereas small interfering RNAs targeting op18 enhanced their sensitivity to GA, suggesting that op18 is a potentially significant target for GA in combating HCC. Results of a study by Chen et al (13) revealed that the upregulation of E2F1 and op18 proteins is associated with worse outcomes in patients with HCC, and E2F1 significantly correlates with the op18 protein level in HCC lesions and in vitro transactivation assays. It was reported that op18 expression may be associated with HCC metastasis, recurrence and prognosis, by detecting op 18 mRNA expression in normal liver, non-metastasis, metastasis and recurrence in HCC tissues.

Our previous findings also suggested that the consecutive upregulation of op18 expression was associated with hepatocarcinogenesis by tissue microarray and IHC technology in normal liver, hepatitis, hepatic cirrhosis and HCC tissue (8). To examine the role of op18 in hepatocarcinogenesis in the present study, we established an op18 overexpression
SMMC7721 cell model and detected the difference in HCC cell proliferation, apoptosis and cell cycle in established cell lines. The results demonstrated that the upregulation of op18 expression induced cell proliferation, inhibited cell apoptosis and arrested the cell cycle from G2 to M phase in SMMC7721 cells, showing that op18 overexpression was closely associated with HCC tumorigenesis.

In an experimental model on HCC and lesions, Singer et al (15) indicated that the overexpression of op 18 correlated with tumor progression, proliferation and activation of a few pro-tumor factors, such as p53. Results of that study demonstrated that op18 expression was associated with HCC cell viability, migration and was mediated by gain-of-function mutations in p53 (15). Accordingly, in an experimental model of large-size HCC mice and in xenograft models of human hepatoma tumors, Chen et al (16) demonstrated an increase in op18-mediated tumor growth by preventing its upregulation of EZH2 gene expression. However, any underlying mechanistic association between op18 expression and hepatocarcinogenesis is poorly understood, suggesting that additional studies are required to investigate the mechanism by which op18 is involved in HCC cell proliferation and apoptosis.

In conclusion, our findings suggest that op18 expression contributes to cell proliferation, represses cell apoptosis and induces cell cycle arrest by inhibiting the progression of G2 to M phase in human HCC SMMC7721-op18 cells. Therefore, op18 is a potential predictor of prognosis in HCC.

\section{Acknowledgements}

This study was supported by the Project of the Sichuan Provincial Department of Education (no. 11ZB125), the Youth Foundation, and the National Natural Science Pre-Research Foundation of Luzhou Medical College (no. 2012ZD-06, 202, 451).

\section{References}

1. Tsai CL, Koong AC, Hsu FM, Graber M, Chen IS and Cheng JC: Biomarker studies on radiotherapy to hepatocellular carcinoma. Oncology 84 (Suppl 1): 64-68, 2013.

2. Psyrri A, Arkadopoulos N, Vassilakopoulou M, Smyrniotis V and Dimitriadis G: Pathways and targets in hepatocellular carcinoma. Expert Rev Anticancer Ther 12: 1347-1357, 2012.

3. Maluccio M and Covey A: Recent progress in understanding, diagnosing, and treating hepatocellular carcinoma. CA Cancer $\mathbf{J}$ Clin 62: 394-399, 2012.

4. D'Andrea S, Berton S, Segatto I, Fabris L, Canzonieri V, Colombatti A, Vecchione A, Belletti B and Baldassarre G: Stathmin is dispensable for tumor onset in mice. PLoS One 7: e45561, 2012.

5. Chen J, Abi-Daoud M, Wang A, Yang X, Zhang X, Feilotter HE and Tron VA: Stathmin 1 is a potential novel oncogene in melanoma. Oncogene 32: 1330-1337, 2013.

6. Tian X, Tian Y, Sarich N, Wu T and Birukova AA: Novel role of stathmin in microtubule-dependent control of endothelial permeability. FASEB J 26: 3862-3874, 2012.

7. Nemunaitis J: Stathmin 1: a protein with many tasks. New biomarker and potential target in cancer. Expert Opin Ther Targets 16: 631-634, 2012.

8. Gan L, Guo K, Li Y, Kang X, Sun L, Shu H and Liu Y: Up-regulated expression of stathmin may be associated with hepatocarcinogenesis. Oncol Rep 23: 1037-1043, 2010.

9. Belletti B and Baldassarre G: Stathmin: a protein with many tasks. New biomarker and potential target in cancer. Expert Opin Ther Targets 15: 1249-1266, 2011. 
10. Long M, Yin G, Liu L, Lin F, Wang X, Ren J, Wei J, Dong K and Zhang H: Adenovirus-mediated Aurora A shRNA driven by stathmin promoter suppressed tumor growth and enhanced paclitaxel chemotherapy sensitivity in human breast carcinoma cells. Cancer Gene Ther 19: 271-281, 2012.

11. Sabherwal Y, Mahajan N, Helseth DL, Gassmann M, Shi H and Zhang M: PDEF downregulates stathmin expression in prostate cancer. Int J Oncol 40: 1889-1899, 2012.

12. Wang X, Chen Y, Han QB, Chan CY, Wang H, Liu Z, Cheng CH, Yew DT, Lin MC, He ML, Xu HX, Sung JJ and Kung HF: Proteomic identification of molecular targets of gambogic acid: role of stathmin in hepatocellular carcinoma. Proteomics 9: 242-253, 2009

13. Chen YL, Uen YH, Li CF, Horng KC, Chen LR, Wu WR, Tseng HY, Huang HY, Wu LC and Shiue YL: The E2F transcription factor 1 transactives stathmin 1 in hepatocellular carcinoma. Ann Surg Oncol 20: 4041-4054, 2013.
14. Hsieh SY, Huang SF, Yu MC, et al: Stathmin1 overexpression associated with polyploidy, tumor-cell invasion, early recurrence, and poor prognosis in human hepatoma. Mol Carcinog 49: 476-487, 2010

15. Singer S, Ehemann V, Brauckhoff A, Keith M, Vreden S, Schirmacher P and Breuhahn K: Protumorigenic overexpression of stathmin/Op18 by gain-of-function mutation in p53 in human hepatocarcinogenesis. Hepatology 46: 759-768, 2007.

16. Chen Y, Lin MC, Yao H, Wang H, Zhang AQ, Yu J, Hui CK, Lau GK, He ML, Sung J and Kung HF: Lentivirus-mediated RNA interference targeting enhancer of zeste homolog 2 inhibits hepatocellular carcinoma growth through down-regulation of stathmin. Hepatology 46: 200-208, 2007. 